Article

\title{
Quartz-Enhanced Photoacoustic Detection of Ethane in the Near-IR Exploiting a Highly Performant Spectrophone
}

\author{
Fabrizio Sgobba ${ }^{1}$, Giansergio Menduni ${ }^{1,2}$, Stefano Dello Russo ${ }^{1}$, Angelo Sampaolo ${ }^{1}$, \\ Pietro Patimisco $^{1}$, Marilena Giglio ${ }^{1} \mathbb{D}$, Ezio Ranieri $^{3}$, Vittorio M. N. Passaro ${ }^{2}{ }^{\mathbb{D}}$, \\ Frank K. Tittel ${ }^{4}(\mathbb{D})$ and Vincenzo Spagnolo ${ }^{1, *}$ (I) \\ 1 PolySense Lab—Dipartimento Interateneo di Fisica, Politecnico and University of Bari, CNR—IFN, \\ Via Amendola 173, I-70126 Bari, Italy; fabrizio.sgobba@uniba.it (F.S.); giansergio.menduni@poliba.it (G.M.); \\ stefano.dellorusso@uniba.it (S.D.R.); angelo.sampaolo@poliba.it (A.S.); pietro.patimisco@uniba.it (P.P.); \\ marilena.giglio@uniba.it (M.G.) \\ 2 Photonics Research Group, Dipartimento di Ingegneria Elettrica e dell'informazione, Politecnico di Bari, \\ Via Orabona 4, 70126 Bari, Italy; vittorio.passaro@poliba.it \\ 3 Dipartimento di Biologia Via Orabona 4, Università di Bari, I-70126 Bari, Italy; ezio.ranieri@uniba.it \\ 4 Department of Electrical and Computer Engineering, Rice University, 6100 Main Street, Houston, TX 77005, \\ USA; fkt@rice.edu \\ * Correspondence: vincenzoluigi.spagnolo@poliba.it
}

Received: 25 February 2020; Accepted: 31 March 2020; Published: 3 April 2020

check for updates

Featured Application: Results obtained in this work are promising tools in order to realize a compact, reliable and robust sensor implementable in portable applications, with particular aim to "unmanned aerial vehicle (UAV)-based sensing".

\begin{abstract}
In this paper the performances of two spectrophones for quartz-enhanced photoacoustic spectroscopy (QEPAS)-based ethane gas sensing were tested and compared. Each spectrophone contains a quartz tuning fork (QTF) acoustically coupled with a pair of micro-resonator tubes and having a fundamental mode resonance frequency of $32.7 \mathrm{kHz}$ (standard QTF) and $12.4 \mathrm{kHz}$ (custom QTF), respectively. The spectrophones were implemented into a QEPAS acoustic detection module (ADM) together with a preamplifier having a gain bandwidth optimized for the respective QTF resonance frequency. Each ADM was tested for ethane QEPAS sensing, employing a custom pigtailed laser diode emitting at $\sim 1684 \mathrm{~nm}$ as the exciting light source. By flowing $1 \%$ ethane at atmospheric pressure, a signal-to-noise ratio of 453.2 was measured by implementing the $12.4 \mathrm{kHz}$ QTF-based $\mathrm{ADM}, \sim 3.3$ times greater than the value obtained using a standard QTF. The minimum ethane concentration detectable using a $100 \mathrm{~ms}$ lock-in integration time achieving the $12.4 \mathrm{kHz}$ custom QTF was $22 \mathrm{ppm}$.
\end{abstract}

Keywords: ethane; quartz-enhanced photoacoustic spectroscopy; laser diode; near-infrared

\section{Introduction}

The number of technological fields requiring in-situ and real time gas tracing is rapidly increasing and consequently the need for ad hoc sensing approaches for specific applications [1,2]. For example, the operating conditions for gas sensors employed in environmental monitoring [3] are very different with respect to sensors implemented in breath analysis systems $[4,5]$ or integrated into pipelines for measuring natural gas composition from downhole [6,7]. In fact, one of the most important challenges in gas sensing is the development of versatile sensors and modules capable of detecting different 
molecular species and operating in a wide range of pressures and temperatures, as well as in harsh environments. Extreme working conditions combined with life-threatening risks, such as intoxication and poisoning of the operators, triggered many remote sensing solutions like the employment of unmanned aerial vehicle (UAVs) [8-10].

Laser based spectroscopic techniques guarantee a high detection selectivity because of the narrow linewidths characterizing the laser source employed in the visible to the terahertz range, capable of exciting the target molecules while not being absorbed by the gas matrix [11-14]. The spectral purity of the excitation source, combined with wavelength/amplitude modulation approaches and the efficiency of the optical detection techniques result in reaching detection sensitivity levels as low as parts per quadrillion [15-17].

Depending on the specific application, these unique characteristics can make laser-based sensors more suitable solutions for remote, fast, selective and sensitive trace gas detections relying on UAVs compared to electrochemical and semiconductor sensors. For instance, a non-dispersive infrared (NDIR) sensor was mounted on a UAV for environmental monitoring and proved to be able to detect $\mathrm{CO}_{2}$ concentrations of $0.5 \%$ in air with a $10 \mathrm{~s}$ warm-up time [8]. Similarly, a tunable diode laser absorption spectroscopy (TDLAS) sensor was mounted on a UAV for remote detection of $\mathrm{CH}_{4}$ clouds and sources localization [18].

Among the laser-based techniques, quartz-enhanced photoacoustic spectroscopy (QEPAS) offers unique advantages, such as the possibility to avoid the use of optical detectors, wavelength-independent detection, compact and robust sensor architectures [19]. The core element of a QEPAS sensor is the spectrophone composed of a quartz tuning fork (QTF) and a pair of micro-resonator tubes. In an on-beam configuration, the QTF is placed between two metallic tubes which are in turn aligned perpendicular to the QTF plane and set at an optimized distance [20]. The laser light is focused between the prongs of the resonator and forced to pass through both tubes without touching them, as otherwise a photothermal background noise affects the sensing performance [21]. In the wavelength modulation approach, the laser light is modulated at half of the resonance frequency of one of the QTF flexural modes and the QEPAS signal is demodulated at the resonance frequency. When the emission wavelength is resonant with an optical transition of the gas target, molecules experience an alternation of optical absorption and non-radiative relaxation processes, giving rise to a pressure wave, i.e., a sound wave. The pressure-induced prongs' vibrations generate a piezo-current proportional to the gas target concentration. The first QTF implemented in a QEPAS sensor was a standard $32.7 \mathrm{kHz}$ QTF designed for timing applications [22]. This QTF has a quality factor of 10,000 in air that increases up to 100,000 in vacuum. Extremely robust and reliable QEPAS sensors implementing standard QTF-based acoustic detection modules (ADM) were demonstrated in literature $[23,24]$ and tested in real world applications [25], and also exploiting the single-mode beam delivery provided by optical fibers in the mid-IR [26,27]. Targeted applications were leak detection [28], hydrocarbon detection [29], and environmental monitoring [30,31].

Starting from 2013, several studies have been performed to design QTFs with custom geometries optimized for QEPAS sensing [20,21,32-41]. An extensive investigation correlated the resonance frequency and the quality factor of the fundamental mode of the QTF with the prong sizes and geometry. It has been shown that if some additional mass is added to the free end of the prong, an increase of the global stress field is obtained, enhancing the stress distribution along the internal prong surface and the QTF sound-to-current transduction efficiency [32,33]. In addition, an enlargement of the prongs spacing of up to $1.5 \mathrm{~mm}$ can facilitate the optical alignment and minimize the photothermal noise level, allowing the use of laser beams characterized by a non-optimum spatial intensity distribution, such as high-power laser beams [34], THz quantum cascade lasers (QCLs) [35], arrays of QCLs [36,37] and dual-antinode configurations [38,39]. The advantages of low-frequency, high-quality factor QTFs with a large prong spacing have been demonstrated in the mid-infrared spectral range, where improvements in the detection limit have been obtained mainly due a the reduction of the photothermal noise contribution. With near infrared diode lasers, the ultimate noise level, namely the QTF Johnson 
noise, has been reached when a standard $32.7 \mathrm{kHz}$ was employed. However, moving to QTFs with large prong spacing can be advantageous also in the near-infrared range. A large prong spacing can avoid misalignments due to external mechanical vibrations, leading to an increase of the robustness, a crucial intended feature for sensors, for example, when mounted on mobile systems or ultimately on a drone.

In this work we report on a comparison between two spectrophones, one implementing a standard $32.7 \mathrm{kHz}$ QTF (SP1) and a second one, a T-shaped $12.4 \mathrm{kHz}$ custom QTF (SP2). Both spectrophones were coupled with a pair of optimized resonator tubes, in an "on beam" configuration. Subsequently they were tested in a QEPAS sensor employing a DFB diode laser emitting at $1684 \mathrm{~nm}$ as a light source in order to target ethane. An investigation on the optimum working conditions as well as an analysis of the ultimate detection limit and long-term stability was also performed.

\section{Resonance Properties of Spectrophones}

The spectrophone SP1 employs a standard $32.7 \mathrm{kHz}$ quartz tuning fork (QTF) coupled with two $4.4 \mathrm{~mm}$ long tubes having inner diameters of $0.6 \mathrm{~mm}$ and positioned $50 \mu \mathrm{m}$ distant from the QTF surface. This resonator tubes configuration has represented the reference spectrophone structure for QEPAS experiments until 2013, allowing a signal-to-noise ratio (SNR) enhancement up to a factor of 30 compared to a bare QTF [19]. Spectrophone SP2 is composed of a QTF with T-shape prongs coupled with two $12.4 \mathrm{~mm}$ long resonator tubes having inner diameters of $1.59 \mathrm{~mm}$ and positioned $200 \mu \mathrm{m}$ distant from the QTF. The prongs spacing is $800 \mu \mathrm{m}, 2.7$ larger than the standard QTF. Enlarging of the prong spacing from $300 \mu \mathrm{m}$ to $800 \mu \mathrm{m}$ allowed the reduction of the $1 \sigma$ noise level by one order of magnitude when operating with mid-infrared quantum cascade laser sources and focusing the optical beam through the spectrophone by using a single lens. A sketch of SP1 and SP2 is shown in Figure 1.

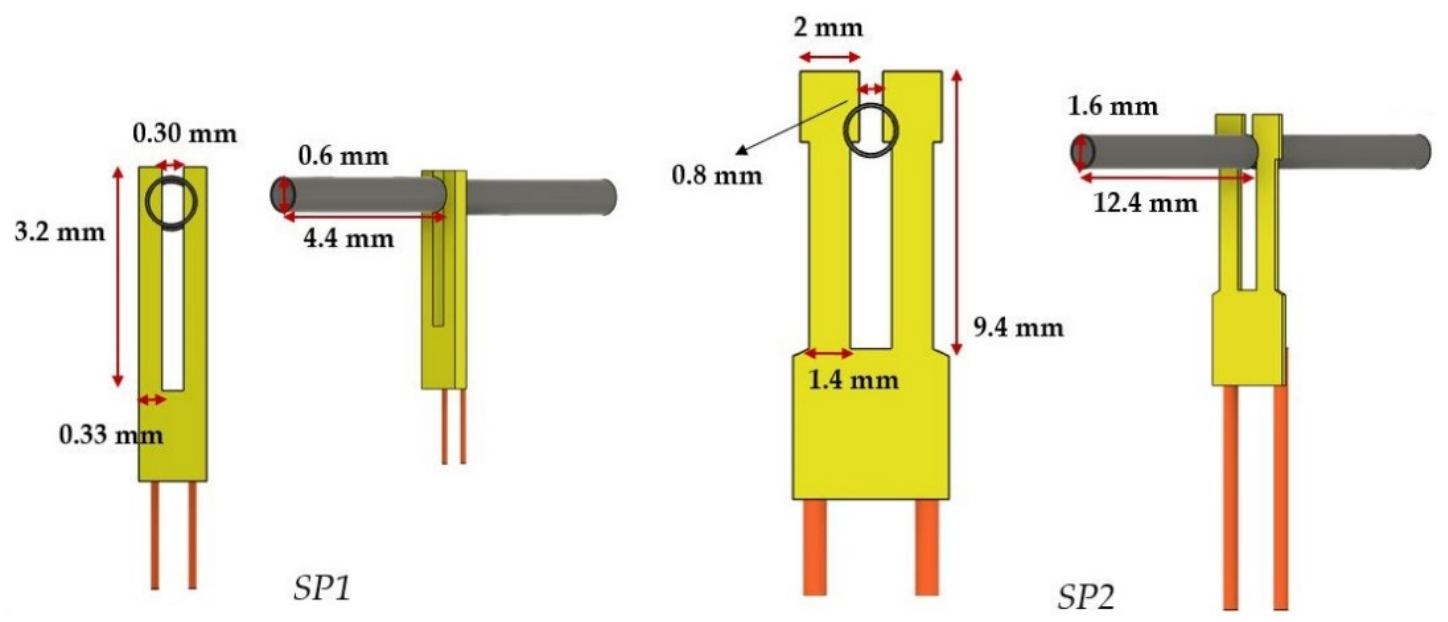

Figure 1. Schematics of spectrophones SP1 (left) and SP2 (right) employed in this article. The main geometrical parameters are also reported.

The acoustic properties of both spectrophones were firstly measured. QTF vibration can be electrically induced by providing a sinusoidal voltage excitation. Thus, the QTF piezo-current can be converted in a voltage signal by means of a transimpedance amplifier and demodulated at the excitation frequency by means of a lock-in amplifier. The SP1 and SP2 spectrophones are mounted in two acoustic detection modules (ADM1 and ADM2, respectively) composed of a vacuum-tight gas cell equipped with two ZnSe wedged $1 / 2$ inch windows, with an antireflection coating in the $1.65-3 \mu \mathrm{m}$ spectral range, and gas-in and gas-out connectors. Each ADM consists of a preamplifier with a gain-bandwidth optimized for the related QTF operating frequency. Resonance curves of both spectrophones can be retrieved by varying the excitation frequency step-by-step. These measurements were performed in air, at atmospheric pressure. The resonance curves were acquired at the respective 
fundamental in-plane flexural mode resonance frequencies and normalized to the peak values are reported in Figure 2.

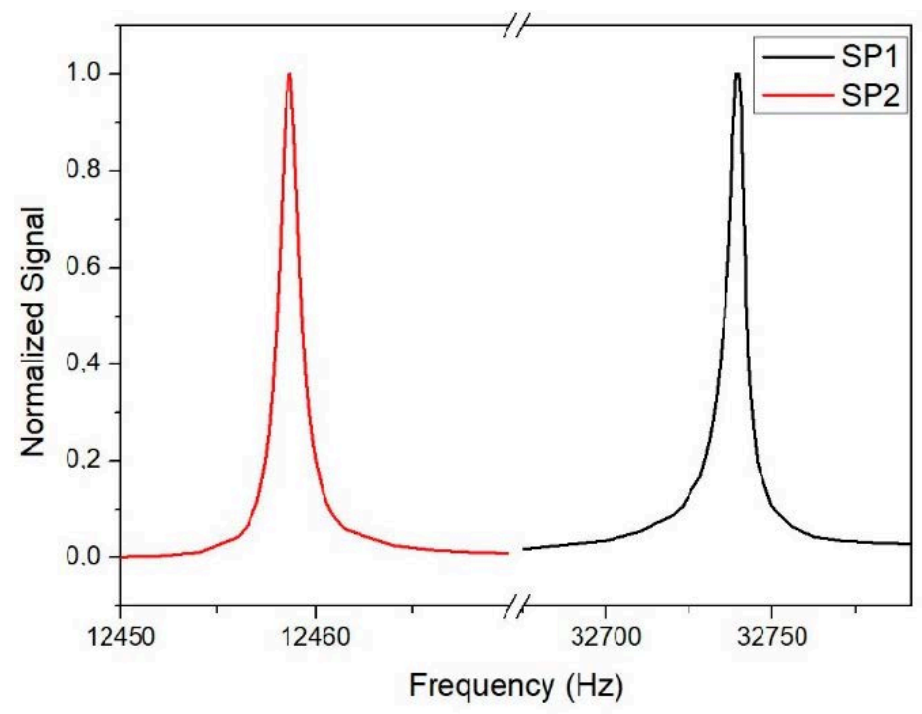

Figure 2. Normalized resonance curves of SP1 (red solid curve) and SP2 (black solid curve) at atmospheric pressure. ADM1 displayed a resonance frequency of 32,738.81 Hz and a quality factor of $\sim 5080$, whereas for ADM2 a resonance frequency of $12,458.67 \mathrm{~Hz}$ and a quality factor of $\sim 9930$ were measured.

To determine the resonance properties of both spectrophones, namely the resonance frequency and the related quality factor, the resonance curves can be fitted by a Lorentzian function. The resonance peak broadening is proportional to the energy losses occurring in the vibrating prongs. Furthermore, the larger the broadening, the higher the losses [40]. The quality factor $Q$ was calculated as the ratio between the resonance frequency and the full-width-half-maximum (FWHM) value of the resonance curve. For ADM1 a resonance frequency of $0.81 \mathrm{~Hz}$ and a quality factor of $\sim 5080$ was measured while SP2 is characterized by a fundamental resonant mode frequency of $0.67 \mathrm{~Hz}$ with a quality factor of 9930. ADM2 exhibits a higher Q-factor with respect to ADM1 despite a lower resonance frequency. Both aspects are beneficial for QEPAS: low resonance frequencies are beneficial for slow relaxing gases and high quality factors positively affecting the QEPAS signal [41].

\section{Ethane QEPAS Sensor}

The architecture of the ethane sensor is depicted in Figure 3.

A distributed-feedback (DFB) pigtailed diode laser emitting at $\sim 1684.5 \mathrm{~nm}$ (Eblana model EP1684-0-DM-B06-FA) was employed as the exciting laser source. Ethane $\left(\mathrm{C}_{2} \mathrm{H}_{6}\right)$ shows an unresolvable vibration-rotational absorption band near $1680 \mathrm{~nm}$ [42-44]. In this spectral range, a well-isolated absorption peak centered at $1684 \mathrm{~nm}$ was identified. A pigtailed single-mode diode laser was coupled with a fiber collimator. The beam profile of the collimated beam was measured by means of a pyroelectric camera with a spatial resolution of $100 \mu \mathrm{m} \times 100 \mu \mathrm{m}$. A beam diameter of $220 \mu \mathrm{m}$ with a divergence of $2 \mathrm{mrad}$ was measured, allowing $\sim 97 \%$ and $~ 99 \%$ of the laser light to pass through ADM1 and ADM2, respectively. A pressure controller, a valve system and a vacuum pump were used to set the pressure inside the gas cell. An MCQ Instruments gas blender model GB100 (not shown in Figure 3) was used to set the gas flow at $50 \mathrm{sccm}$. Measurements were performed using wavelength modulation and 2f-detection: a sinusoidal dither at half of the spectrophone resonance frequency was applied to the laser current driver using a waveform generator and the QTF signal was demodulated at the spectrophone resonance frequency by a lock-in amplifier. QEPAS spectra were obtained by adding a voltage triangle-shaped ramp to the laser fast modulation current in order to scan the laser 
wavelength across the ethane absorption line. The lock-in integration time was set at $100 \mathrm{~ms}$, while the acquisition time was set at $300 \mathrm{~ms}$.

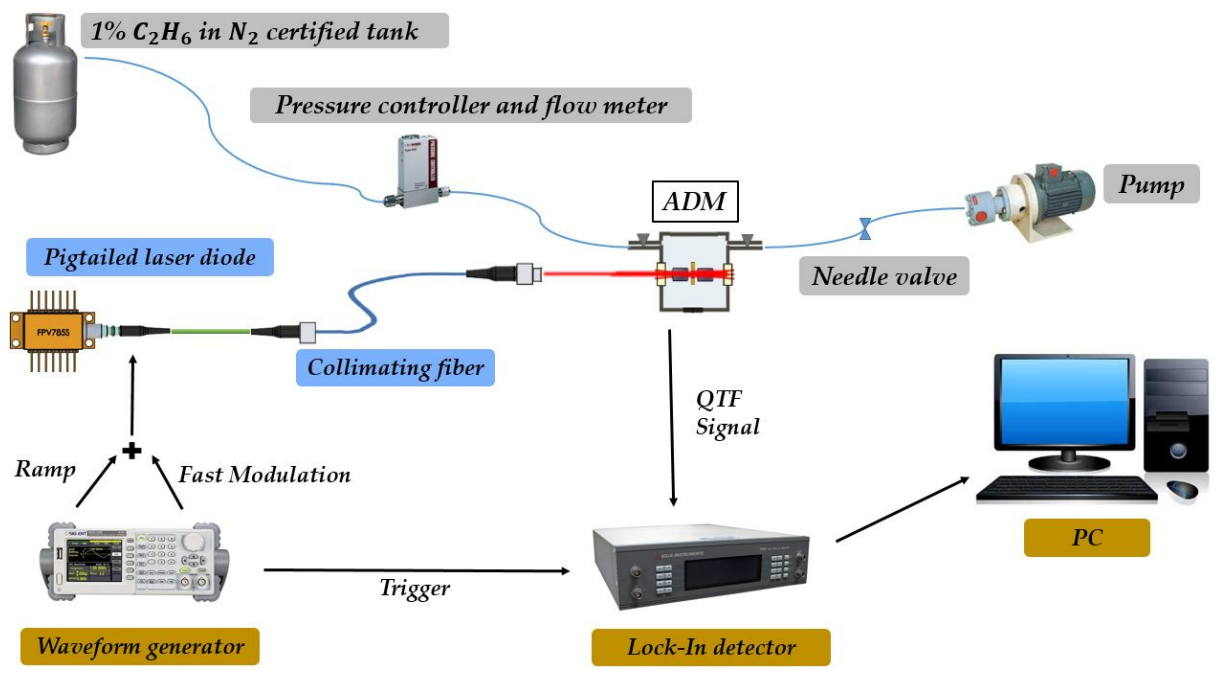

Figure 3. Schematic of the QEPAS sensor for ethane detection. ADM: acoustic detection module. The experimental setup was conceived in order to easily swap the two ADMs while keeping fixed the rest of the apparatus. The light blue curves mimic the gas line.

\section{Results}

The performance of QEPAS sensors depends on the gas pressure. High QTF quality factors and efficient sounds generation are required for QEPAS operation. When reducing the pressures ( $<200$ Torr), the QTF quality factor increases while the number of molecules diminishes, negatively affecting the sound generation efficiency. Thereby an optimal gas pressure maximizing the QEPAS signal, as tradeoff between two opposite trends, has to be identified.

A certified $1 \% \mathrm{C}_{2} \mathrm{H}_{6}: \mathrm{N}_{2}$ gas mixture was used to investigate the QEPAS signal as a function of the pressure, for both spectrophones. The current amplitude modulation depends on the gas pressure since the full width half maximum (FWHM) of the absorption line suffers from the pressure broadening. Similarly, the resonance frequency of the spectrophone is pressure-dependent: when prongs vibrate in a viscous fluid. This effect can be modeled as an increase of the prong's effective mass, which corresponds to a linear decrease of the resonance frequency while the pressure increases. Thus, for each spectrophone and for each pressure value the current amplitude modulation and the resonance frequency of the spectrophone were optimized. Ethane QEPAS peak values, occurring at an injection current of $112.5 \mathrm{~mA}$ and a TEC temperature of $16^{\circ} \mathrm{C}$, are reported in Figure 4 as a function of gas pressure for both spectrophones.

Data were normalized to the peak value corresponding to an optimum pressure in order to make the correlation between excitation frequency (represented by the different resonator) and gas pressure independent of extrinsic factors such as the employed preamplifier.

This preliminary investigation allowed identification of the best operating condition of the two spectrophones for ethene detection, namely pressure, waveform amplitude and frequency modulation of 300 Torr, $125 \mathrm{mV}$ and $0.49 \mathrm{~Hz}$ for SP1 and 750 Torr, $150 \mathrm{mV}$ and $6229.43 \mathrm{~Hz}$ for SP2. The performances of the sensors employing the two ADMs operating at their best conditions are compared in Figure 5, where the QEPAS spectral scans at the ethane peak are reported. 


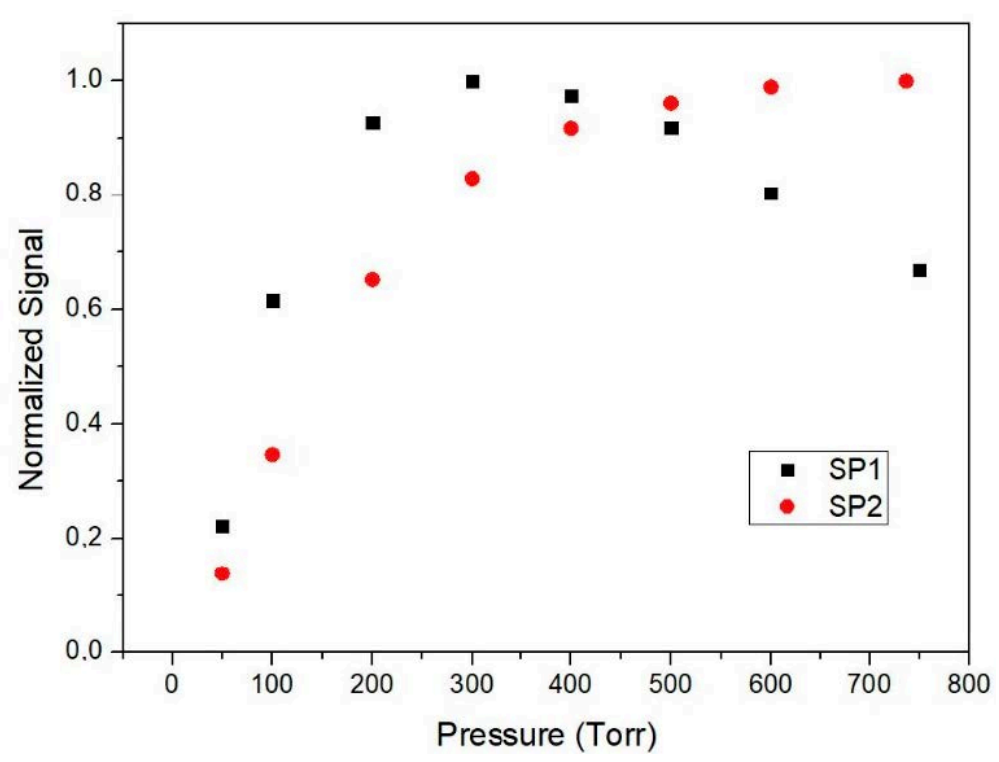

Figure 4. Normalized QEPAS peak signals at fixed ethane concentration ( $1 \%$ in nitrogen, certified) as a function of pressure measured for SP1 (black squares) and SP2 (red dots).

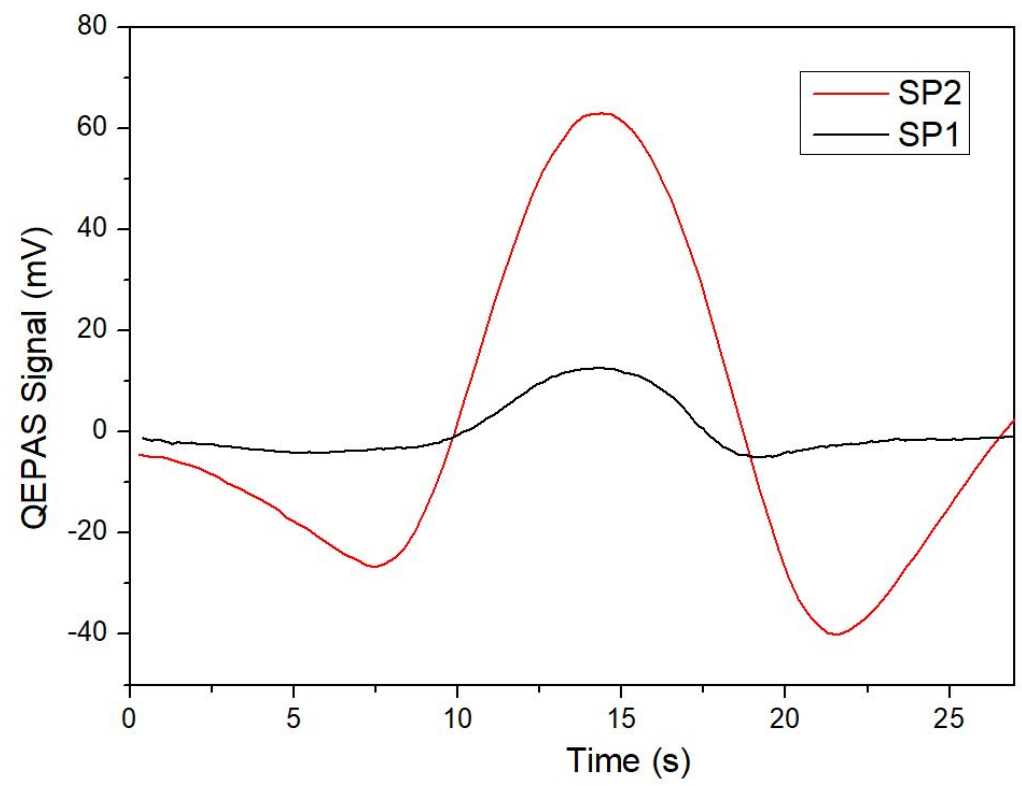

Figure 5. Spectral scan of the ethane peak with QEPAS sensor employing SP1 (black curve) and SP2 (red curve).

Figure 5 depicts the measured ethane QEPAS signals, resembling the opposite of the second derivative of the ethane absorption Lorentzian line-shape [41]. The peak is reached at $\sim 15 \mathrm{~s}$ time of acquisition, corresponding to a wavelength of $1684 \mathrm{~nm}\left(5937.3 \mathrm{~cm}^{-1}\right)$. The peak signal measured with the ADM2 is $63.0 \mathrm{mV}$, 4.9 times higher than the $12.9 \mathrm{mV}$-peak signal measured by implementing ADM1. Using an averaging time of $100 \mathrm{~ms}$, a $1-\sigma$ noise of $0.094 \mathrm{mV}$ for ADM1 and $0.139 \mathrm{mV}$ for ADM2 was measured. This gives signal-to-noise ratios and minimum detection limits (MDL) of: SNR1 136, MDL1 $\sim 73$ ppm and SNR2 453, MDL2 22 ppm, respectively for ADM1 and ADM2. The sensor long-term stability was then investigated. A $30 \mathrm{~min}$ long acquisition of consecutive QEPAS spectral scans of the ethane absorption line was performed employing each ADM at the best-found operating conditions. The peak signal value of each ramp, extracted by means of a MATLAB software, was plotted in Figure 6. Repeated scan-measurements were preferred to long-term acquisitions locked on a peak signal in order to neglect possible laser wavelength and optical power instabilities. The peak values recorded over the 
time are plotted in Figure 6. The ethane QEPAS sensor stability was investigated using both ADMs. Peak signal fluctuations of $0.193 \mathrm{mV}$ and $0.277 \mathrm{mV}$ were retrieved for SP1 and SP2, respectively. These fluctuations are higher than the noise level for both ADMs. However, the certified gas mixture of $1 \%$ $\mathrm{C}_{2} \mathrm{H}_{6}: \mathrm{N}_{2}$ flowing into the ADM using the gas mixture has an uncertainty of $1 \%$ on the concentration value, corresponding to a fluctuation of $100 \mathrm{ppm}$ in ethane concentration. The signal fluctuations expected from a 100-ppm gas concentration uncertainty are $\Delta \mathrm{C} 1=0.129 \mathrm{mV}$ for SP1 and $\Delta \mathrm{C} 2=$ $0.630 \mathrm{mV}$ for SP2. Thereby, the standard deviation expected considering the 1- $\sigma$ noise level and gas concentration fluctuations results in $\Delta \mathrm{S} 1=0.160 \mathrm{mV}$ for SP1 and $\Delta \mathrm{S} 2=0.645 \mathrm{mV}$ for SP2. Comparing $\Delta \mathrm{S} 1$ and $\Delta \mathrm{S} 2$ with the measured peak signal fluctuations, it can be observed that the noise fluctuations measured using the T-Shaped QTF spectrophone are lower than expected, denoting a better mechanical stability of S08-T based sensor (SP2) with respect to the standard QTF based one (SP1).

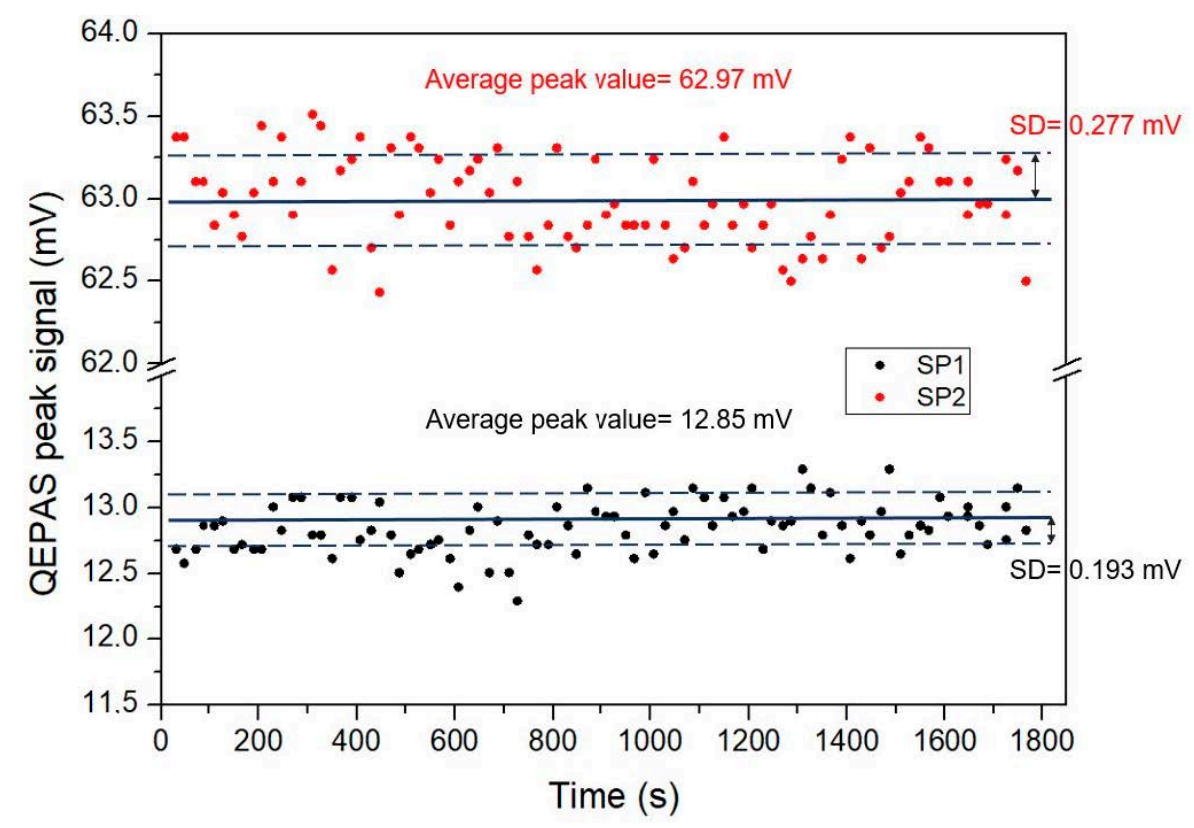

Figure 6. QEPAS peaks trend as a function of the acquisition time, obtained by peak extraction in a continuous spectral scan acquisition.

Once determined the most performing spectrophone in terms of SNR, the linearity of SP2 photoacoustic response over a wide range of ethane concentrations in pure $\mathrm{N}_{2}$ was verified. The gas line depicted in Figure 3 was modified in order to connect a pure $\mathrm{N}_{2}$ gas tank to a separate flow controller of the gas blender to obtain different dilutions starting from the certified concentration of $1 \%$ $\mathrm{C}_{2} \mathrm{H}_{6}: \mathrm{N}_{2}$ down to $100 \mathrm{ppm} \mathrm{C}_{2} \mathrm{H}_{6}: \mathrm{N}_{2}$. The measurements reported in Figure 7 were performed at the same experimental conditions adopted to acquire the $2 \mathrm{f}$-signal scan of the ethane absorption shown in Figure 5 for the SP2 spectrophone.

As can be seen from Figure 7, the QEPAS signal acquired with SP2 spectrophone shows a linear dependence on ethane concentration over two orders of magnitude, from $100 \mathrm{ppm}$ up to $1 \%$, with a slope of $0.00627 \mathrm{mV} / \mathrm{ppm}$. 


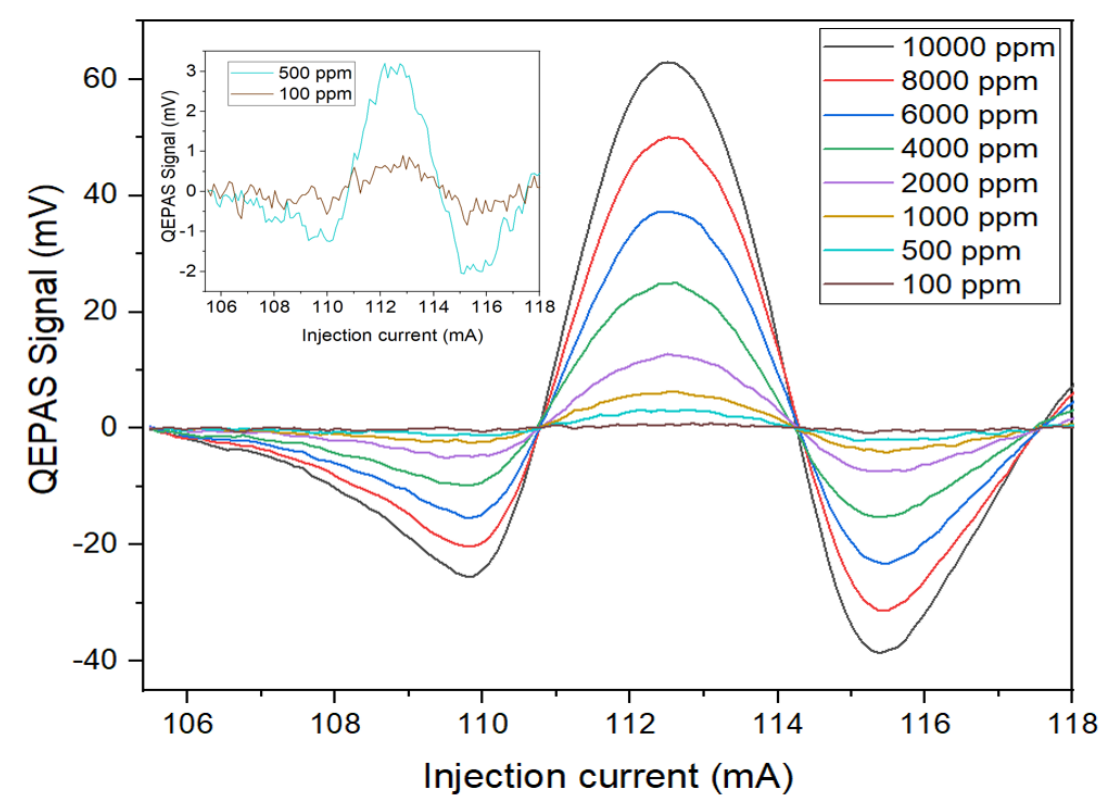

Figure 7. Spectral scan of the ethane absorption line for eight different concentrations of $\mathrm{C}_{2} \mathrm{H}_{6}: \mathrm{N}_{2}$, in the range $100 \mathrm{ppm}-1 \%$, plotted as a function of injected current. In the inset, a detail of the spectra acquired for $100 \mathrm{ppm}$ and $500 \mathrm{ppm}_{2} \mathrm{H}_{6}: \mathrm{N}_{2}$ concentrations are shown.

\section{Conclusions and Outlook}

In this manuscript the performances of two acoustic detection modules for the detection of ethane in the near IR range $(1684 \mathrm{~nm})$ have been compared. The two ADMs are composed of a spectrophone and a current-to-voltage transducer preamplifier having a gain-bandwidth optimized for the resonance frequency of the employed QTF. The two spectrophones employ a standard $32.7 \mathrm{kHz}$ quartz tuning fork (SP1) and a custom $12.4 \mathrm{kHz}$ quartz tuning fork (SP2), respectively, acoustically coupled with a pair of micro-resonator tubes. For each ADM a preliminary test allowed identifying the best operating conditions. As already demonstrated in the mid-IR range as well as in the near-IR range operating with custom QTFs is preferable. Indeed, SP2 demonstrated a better QEPAS sensor long-term stability with respect to SP1. Furthermore, the SNR measured using SP2 was 3.3 times greater than the value measured using SP1, thus leading to a lower minimum detection limit. The linearity of photoacoustic detection was also proven for SP2 spectrophone over a wide $\mathrm{C}_{2} \mathrm{H}_{6}$ concentration range, from $100 \mathrm{ppm}$ up to $1 \%$, with a conversion factor of $0.00627 \mathrm{mV} / \mathrm{ppm}$. The sensor presented in this work represents the first step for the development of a prototype lightweight, low-power consumption, high-sensitivity, robust sensor system, allowing installation of the sensor on UAVs and real-time in situ ethane gas leak detection.

Author Contributions: Conceptualization, all (F.S., G.M., S.D.R., A.S., P.P., M.G., E.R., V.M.N.P., F.K.T. and V.S.); methodology, All; investigation, F.S., G.M., A.S., P.P. and M.G.; data curation, All; writing-original draft preparation, F.S. and G.M.; writing-review and editing, All; supervision, V.S. All authors have read and agreed to the published version of the manuscript.

Funding: The authors from Dipartimento Interateneo di Fisica di Bari acknowledge the financial support from the European Union's Horizon 2020 research and innovation program under the Marie Skłodowska-Curie project OPTAPHI, grant No. 860808 and from THORLABS GmbH within the joint-research laboratory. Frank K. Tittel acknowledges support by the Robert Welch Foundation (Grant No. C0586).

Acknowledgments: Authors acknowledge Eblana Photonics for the design and realization of a custom pigtailed laser diode having center emission wavelength of $1684 \mathrm{~nm}$.

Conflicts of Interest: The authors declare no conflict of interest. 


\section{References}

1. Liu, X.; Cheng, S.; Liu, H.; Hu, S.; Zhang, D.; Ning, H. A survey on gas sensing technology. Sensors 2012, 12, 9635-9665. [CrossRef]

2. Hodgkinson, J.; Tatam, R.P. Optical gas sensing: A review. Meas. Sci. Technol. 2013, 24, 012004. [CrossRef]

3. Wu, T.; Chen, W.; Fertein, E.; Cazier, F.; Dewaele, D.; Gao, X. Development of an open-path incoherent broadband cavity-enhanced spectroscopy based instrument for simultaneous measurement of HONO and NO2 in ambient air. Appl. Phys. B 2012, 106, 501-509. [CrossRef]

4. Fortes, P.R. Optical Gas Sensors for Exhaled Breath Analysis; SPIE: Bellingham, WA, USA, 2017; ISBN 978-1-5106-1378-2.

5. Parameswaran, K.R.; Rosen, D.I.; Allen, M.G.; Ganz, A.M.; Risby, T.H. Off-axis integrated cavity output spectroscopy with a mid-infrared interband cascade laser for real-time breath ethane measurements. Appl. Opt. 2009, 48, B73-B79. [CrossRef] [PubMed]

6. Florence, F.R.; Burks, J. New surface and down-hole sensors needed for oil and gas drilling. In Proceedings of the 2012 IEEE International Instrumentation and Measurement Technology Conference Proceedings, Graz, Austria, 13-16 May 2012; IEEE: Piscataway, NJ, USA, 2012; pp. 670-675.

7. Christopher, M.J.; Freese, R.; Perkins, D.; Dai, B. Photonics Applied: Energy: Multivariate Optical Computing Enables Accurate Harsh-Environment Sensing for the Oil and Gas Industry; Laser Focus World, PennWell Corporation: Tulsa, OK, USA, 2014; pp. 27-31.

8. Kersnovski, T.; Gonzalez, F.; Morton, K. A UAV system for autonomous target detection and gas sensing. In Proceedings of the 2017 IEEE Aerospace Conference, Big Sky, MT, USA, 4-11 March 2017; IEEE: Piscataway, NJ, USA, 2017; pp. 1-12.

9. Rossi, M.; Brunelli, D.; Adami, A.; Lorenzelli, L.; Menna, F.; Remondino, F. Gas-Drone: Portable gas sensing system on UAVs for gas leakage localization. In Proceedings of the IEEE Sensors 2014 Proceedings, Valencia, Spain, 2-5 November 2014; IEEE: Piscataway, NJ, USA, 2014; pp. 1431-1434.

10. Rossi, M.; Brunelli, D. Gas sensing on unmanned vehicles: Challenges and opportunities. In Proceedings of the 2017 New Generation of CAS (NGCAS), Genova, Italy, 6-9 September 2017; IEEE: Piscataway, NJ, USA, 2017; pp. 117-120.

11. Liu, K.; Zha, S.; Liu, Q.; Chen, W.; Xiaoming, G. Development of photoacoustic spectroscopy sensor for aerosol optical absorption measurement. In Proceedings of the Light, Energy and the Environment 2015, Suzhou, China, 2-5 November 2015; OSA: Washington, DC, USA, 2015; p. JTu5A.18.

12. Phillips, M.C.; Myers, T.L.; Wojcik, M.D.; Cannon, B.D. External cavity quantum cascade laser for quartz tuning fork photoacoustic spectroscopy of broad absorption features. Opt. Lett. 2007, 32, 1177-1179. [CrossRef]

13. Glauvitz, N.E.; Coutu, R.A.; Medvedev, I.R.; Petkie, D.T. Terahertz photoacoustic spectroscopy using an MEMS cantilever sensor. J. Microelectromech. Syst. 2015, 24, 216-223. [CrossRef]

14. Zhu, X.; Yao, S.; Ren, W.; Lu, Z.; Li, Z. TDLAS monitoring of carbon dioxide with temperature compensation in power plant exhausts. Appl. Sci. 2019, 9, 442. [CrossRef]

15. Xiong, L.; Bai, W.; Chen, F.; Zhao, X.; Yu, F.; Diebold, G.J. Photoacoustic trace detection of gases at the parts-per-quadrillion level with a moving optical grating. Proc. Natl. Acad. Sci. USA 2017, 114, 7246-7249. [CrossRef]

16. Galli, I.; Bartalini, S.; Ballerini, R.; Barucci, M.; Cancio, P.; De Pas, M.; Giusfredi, G.; Mazzotti, D.; Akikusa, N.; De Natale, P. Spectroscopic detection of radiocarbon dioxide at parts-per-quadrillion sensitivity. Optica 2016, 3, 385-388. [CrossRef]

17. Tomberg, T.; Vainio, M.; Hieta, T.; Halonen, L. Sub-parts-per-trillion level sensitivity in trace gas detection by cantilever-enhanced photo-acoustic spectroscopy. Sci. Rep. 2018, 8, 1848. [CrossRef]

18. Neumann, P.P.; Kohlhoff, H.; Hullmann, D.; Lilienthal, A.J.; Kluge, M. Bringing mobile robot olfaction to the next dimension-UAV-based remote sensing of gas clouds and source localization. In Proceedings of the 2017 IEEE International Conference on Robotics and Automation (ICRA), Singapore, 29 May-3 June 2017; IEEE: Piscataway, NJ, USA, 2017; pp. 3910-3916.

19. Patimisco, P.; Sampaolo, A.; Dong, L.; Tittel, F.K.; Spagnolo, V. Recent advances in quartz enhanced photoacoustic sensing. Appl. Phys. Rev. 2018, 5, 011106. [CrossRef] 
20. Dello Russo, S.; Giglio, M.; Sampaolo, A.; Patimisco, P.; Menduni, G.; Wu, H.; Dong, L.; Passaro, V.M.N.; Spagnolo, V. Acoustic coupling between resonator tubes in quartz-enhanced photoacoustic spectrophones employing a large prong spacing tuning fork. Sensors 2019, 19, 4109. [CrossRef] [PubMed]

21. Patimisco, P.; Sampaolo, A.; Zheng, H.; Dong, L.; Tittel, F.K.; Spagnolo, V. Quartz-enhanced photoacoustic spectrophones exploiting custom tuning forks: A review. Adv. Phys. X 2017, 2, 169-187. [CrossRef]

22. Kosterev, A.A.; Bakhirkin, Y.A.; Curl, R.F.; Tittel, F.K. Quartz-enhanced photoacoustic spectroscopy. Opt. Lett. 2002, 27, 1902-1904. [CrossRef]

23. Zheng, H.; Dong, L.; Yin, X.; Liu, X.; Wu, H.; Zhang, L.; Ma, W.; Yin, W.; Jia, S. Ppb-level QEPAS NO2 sensor by use of electrical modulation cancellation method with a high power blue LED. Sens. Actuators B Chem. 2015, 208, 173-179. [CrossRef]

24. Zheng, H.; Dong, L.; Ma, Y.; Wu, H.; Liu, X.; Yin, X.; Zhang, L.; Ma, W.; Yin, W.; Xiao, L.; et al. Scattered light modulation cancellation method for sub-ppb-level $\mathrm{NO}_{2}$ detection in a LD-excited QEPAS system. Opt. Express 2016, 24, A752-A761. [CrossRef]

25. Zheng, H.; Dong, L.; Wu, H.; Yin, X.; Xiao, L.; Jia, S.; Curl, R.F.; Tittel, F.K. Application of acoustic micro-resonators in quartz-enhanced photoacoustic spectroscopy for trace gas analysis. Chem. Phys. Lett. 2018, 691, 462-472. [CrossRef]

26. Sampaolo, A.; Patimisco, P.; Kriesel, J.M.; Tittel, F.K.; Scamarcio, G.; Spagnolo, V. Single mode operation with mid-IR hollow fibers in the range 51-105 $\mu \mathrm{m}$. Opt. Express 2015, 23, 195-204. [CrossRef]

27. Giglio, M.; Patimisco, P.; Sampaolo, A.; Kriesel, J.M.; Tittel, F.K.; Spagnolo, V. Low-loss and single-mode tapered hollow-core waveguides optically coupled with interband and quantum cascade lasers. Opt. Eng. 2017, 57, 011004.

28. Sampaolo, A.; Patimisco, P.; Giglio, M.; Chieco, L.; Scamarcio, G.; Tittel, F.K.; Spagnolo, V. Highly sensitive gas leak detector based on a quartz-enhanced photoacoustic SF6 sensor. Opt. Express 2016, 24, 15872-15881. [CrossRef]

29. Sampaolo, A.; Csutak, S.; Patimisco, P.; Giglio, M.; Menduni, G.; Passaro, V.; Tittel, F.K.; Deffenbaugh, M.; Spagnolo, V. Methane, ethane and propane detection using a compact quartz enhanced photoacoustic sensor and a single interband cascade laser. Sens. Actuators B Chem. 2019, 282, 952-960. [CrossRef]

30. He, Y.; Ma, Y.; Tong, Y.; Yu, X.; Tittel, F.K. A portable gas sensor for sensitive CO detection based on quartz-enhanced photoacoustic spectroscopy. Opt. Laser Technol. 2019, 115, 129-133. [CrossRef]

31. He, Y.; Ma, Y.; Tong, Y.; Yu, X.; Tittel, F.K. HCN ppt-level detection based on a QEPAS sensor with amplified laser and a miniaturized 3D-printed photoacoustic detection channel. Opt. Express 2018, 26, 9666-9675. [CrossRef] [PubMed]

32. Patimisco, P.; Sampaolo, A.; Giglio, M.; dello Russo, S.; Mackowiak, V.; Rossmadl, H.; Cable, A.; Tittel, F.K.; Spagnolo, V. Tuning forks with optimized geometries for quartz-enhanced photoacoustic spectroscopy. Opt. Express 2019, 27, 1401-1415. [CrossRef]

33. Giglio, M.; Elefante, A.; Patimisco, P.; Sampaolo, A.; Sgobba, F.; Rossmadl, H.; Mackowiak, V.; Wu, H.; Tittel, F.K.; Dong, L.; et al. Quartz-enhanced photoacoustic sensor for ethylene detection implementing optimized custom tuning fork-based spectrophone. Opt. Express 2019, 27, 4271-4280. [CrossRef]

34. Wu, H.; Sampaolo, A.; Dong, L.; Patimisco, P.; Liu, X.; Zheng, H.; Yin, X.; Ma, W.; Zhang, L.; Yin, W.; et al. Quartz enhanced photoacoustic $\mathrm{H}_{2} \mathrm{~S}$ gas sensor based on a fiber-amplifier source and a custom tuning fork with large prong spacing. Appl. Phys. Lett. 2015, 107, 111104. [CrossRef]

35. Sampaolo, A.; Patimisco, P.; Giglio, M.; Vitiello, M.; Beere, H.; Ritchie, D.; Scamarcio, G.; Tittel, F.; Spagnolo, V. Improved tuning fork for terahertz quartz-enhanced photoacoustic spectroscopy. Sensors 2016, 16, 439. [CrossRef]

36. Giglio, M.; Patimisco, P.; Sampaolo, A.; Zifarelli, A.; Blanchard, R.; Pfluegl, C.; Witinski, M.F.; Vakhshoori, D.; Tittel, F.K.; Spagnolo, V. Nitrous oxide quartz-enhanced photoacoustic detection employing a broadband distributed-feedback quantum cascade laser array. Appl. Phys. Lett. 2018, 113, 171101. [CrossRef]

37. Giglio, M.; Zifarelli, A.; Sampaolo, A.; Menduni, G.; Elefante, A.; Blanchard, R.; Pfluegl, C.; Witinski, M.F.; Vakhshoori, D.; Wu, H.; et al. Broadband detection of methane and nitrous oxide using a distributed-feedback quantum cascade laser array and quartz-enhanced photoacoustic sensing. Photoacoustics 2020, 17, 100159. [CrossRef] 
38. Elefante, A.; Giglio, M.; Sampaolo, A.; Menduni, G.; Patimisco, P.; Passaro, V.M.N.; Wu, H.; Rossmadl, H.; Mackowiak, V.; Cable, A.; et al. Dual-gas quartz-enhanced photoacoustic sensor for simultaneous detection of methane/nitrous oxide and water vapor. Anal. Chem. 2019, 91, 12866-12873. [CrossRef]

39. Zheng, H.; Dong, L.; Patimisco, P.; Wu, H.; Sampaolo, A.; Yin, X.; Li, S.; Ma, W.; Zhang, L.; Yin, W.; et al. Double antinode excited quartz-enhanced photoacoustic spectrophone. Appl. Phys. Lett. 2017, 110, 021110. [CrossRef]

40. Giglio, M.; Menduni, G.; Patimisco, P.; Sampaolo, A.; Elefante, A.; Passaro, V.M.N.; Spagnolo, V. Damping mechanisms of piezoelectric quartz tuning forks employed in photoacoustic spectroscopy for trace gas sensing. Phys. Status Solidi A 2019, 216, 1800552. [CrossRef]

41. Patimisco, P.; Scamarcio, G.; Tittel, F.; Spagnolo, V. Quartz-enhanced photoacoustic spectroscopy: A review. Sensors 2014, 14, 6165-6206. [CrossRef] [PubMed]

42. Hepp, M.; Herman, M. Vibration-rotation bands in ethane. Mol. Phys. 2000, 98, 57-61. [CrossRef]

43. Smith, L.G. The infra-red spectrum of $\mathrm{C}_{2} \mathrm{H}_{6}$. J. Chem. Phys. 1949, 17, 139-167. [CrossRef]

44. Tian, X.; Cao, Y.; Chen, J.; Liu, K.; Wang, G.; Tan, T.; Mei, J.; Chen, W.; Gao, X. Dual-gas sensor of $\mathrm{CH}_{4} / \mathrm{C}_{2} \mathrm{H}_{6}$ based on wavelength modulation spectroscopy coupled to a home-made compact dense-pattern multipass cell. Sensors 2019, 19, 820. [CrossRef]

(C) 2020 by the authors. Licensee MDPI, Basel, Switzerland. This article is an open access article distributed under the terms and conditions of the Creative Commons Attribution (CC BY) license (http://creativecommons.org/licenses/by/4.0/). 